\title{
Is pathological T4a an independent indication of adjuvant therapy in buccal mucosal or gingival squamous cell carcinoma?
}

\author{
Tzu-Yuan Chao $^{1} \wedge$, Leong-Perng Chan ${ }^{2,3,4}$, Hsin-Hua Lee ${ }^{1,5,6,7} \wedge$, Yu-Hsun Kao ${ }^{8}$, Ying-Pei Jhong ${ }^{1 \wedge}$, \\ Chen-Han Chiang $^{1 \wedge}$, Shu-Ting Chuang ${ }^{1 \wedge}$, Chih-Jen Huang ${ }^{1,5,6}$
}

${ }^{1}$ Department of Radiation Oncology, Kaohsiung Medical University Hospital, Kaohsiung City, Taiwan; ${ }^{2}$ Department of OtorhinolaryngologyHead and Neck Surgery, Kaohsiung Municipal Ta-Tung Hospital and Kaohsiung Medical University Hospital, Kaohsiung City, Taiwan; ${ }^{3}$ Cohort Research Center, Kaohsiung Medical University, Kaohsiung City, Taiwan; ${ }^{4}$ Department of Otorhinolaryngology-Head and Neck Surgery, Faculty of Medicine, College of Medicine, Kaohsiung Medical University Hospital, Kaohsiung City, Taiwan; ${ }^{5}$ Center for Cancer Research, Kaohsiung Medical University Hospital, Kaohsiung City, Taiwan; ${ }^{6}$ Department of Radiation Oncology, Faculty of Medicine, College of Medicine, Kaohsiung Medical University Hospital, Kaohsiung City, Taiwan; ${ }^{7} \mathrm{Ph} . \mathrm{D}$. Program in Environmental and Occupational Medicine, Kaohsiung Medical University and National Health Research Institutes, Kaohsiung City, Taiwan; ${ }^{8}$ Division of Oral Maxillofacial Surgery, Department of Dentistry, Kaohsiung Medical University Hospital, Kaohsiung City, Taiwan

Contributions: (I) Conception and design: TY Chao, LP Chan, CJ Huang; (II) Administrative support: TY Chao, HH Lee, CJ Huang; (III) Provision of study materials or patients: LP Chan, YH Kao, CJ Huang; (IV) Collection and assembly of data: TY Chao, HH Lee, ST Chuang, CJ Huang; (V) Data analysis and interpretation: YP Jhong, CH Chiang; (VI) Manuscript writing: All authors; (VII) Final approval of manuscript: All authors.

Correspondence to: Chih-Jen Huang, MD, PhD. Department of Radiation Oncology, Kaohsiung Medical University Hospital, No.100, Tzyou 1st Rd., Sanmin Dist., Kaohsiung City 80756, Taiwan. Email: ccjjhh@kmu.edu.tw.

Background: To evaluate the clinicopathologic factors affecting the clinical outcomes in pathological T4aN0 (pT4aN0) buccal mucosal or gingival squamous cell carcinoma (SCC).

Methods: This was a retrospective study of 113 consecutive patients with diagnoses of pT4aN0 buccal mucosal or gingival SCC between January 2010 and November 2018. The median follow-up was 38 months (range, 1-119 months). Kaplan-Meier and Cox proportional-hazards models were used for survival analysis. Results: In the entire study, 5-year overall survival (OS) and disease-free survival (DFS) rates were $73.7 \%$ and $83.6 \%$, respectively. Univariate analysis revealed that lymphovascular invasion (LVI), perineural invasion (PNI), and margin status had significant effects on the DFS rate, and tumor cell differentiation had a significant effect on OS rate. Multivariate Cox regression analysis revealed margin status was the only significant factor affecting DFS. The patients $(n=38)$ without the inadequate margin, LVI, PNI, and poorly differentiated tumor cells had a better 5-year DFS rate $(\mathrm{P}=0.023)$ whether they had received adjuvant treatment or not (91.5\% versus $100 \%)$. However, no significant effect on the OS rate was found (P=0.544).

Conclusions: The patients who had no adverse pathologic factors exhibited fair DFS rate without adjuvant therapy. The adjuvant therapy may not be considered for this subgroup.

Keywords: Oral cavity squamous cell carcinoma (OCSCC); pathological T4aN0 (pT4aN0); buccal cancer; gingival cancer; adjuvant radiotherapy

Received: 18 February 2021; Accepted: 25 June 2021; Published: 30 September 2021.

doi: $10.21037 /$ tro-21-1

View this article at: https://dx.doi.org/10.21037/tro-21-1

\footnotetext{
^ ORCID: Tzu-Yuan Chao, 0000-0002-3336-4877; Hsin-Hua Lee, 0000-0001-5655-4641; Ying-Pei Jhong, 0000-0002-4816-7809; Chen-Han Chiang, 0000-0002-9721-0404.
} 


\section{Introduction}

In Taiwan, approximately 5,000 new oral cavity squamous cell carcinoma (OCSCC) cases were recorded, accounting for more than half of all head and neck cancer cases, according to the Taiwan Cancer Registry Annual Report, 2017 (1). Many Taiwanese patients with OCSCC have tumors arising from the buccal mucosa or gingival subsite, whereas the prevalence is significantly lower in Western countries (2). This phenomenon has been attributed to the well-known carcinogenic effect of betel quid chewing and smoking (3). In clinical practice, these patients often present with locally advanced cancer diagnosed as stage IV. In the American Joint Committee on Cancer (AJCC) staging (4), stage IV is divided into IVA, IVB, and IVC, with stage IVA accounting for the greatest proportion of cases. This group features much diversity and heterogeneity such as various etiological and subsite locations (5).

The outcomes of pathological T4aN0 (pT4aN0) buccal or gingival squamous cell carcinoma (SCC) have been minimally reported, although these patients' outcomes are often superior to those of patients with other tumor and nodal classifications in stage IVA. The tumor invades the surrounding tissue early in the development of the disease and these groups may be upstaged to pT4a. Currently, surgical intervention followed by adjuvant treatment is the gold standard treatment for OCSCC with unfavorable clinical or pathological factors or both $(6,7)$. However, an increasing number of patients' diseases are not recurrent during long-term follow-up without any adjuvant treatment. In our study, our objective was to identify factors predicting the clinical outcomes of patients with pT4aN0 buccal mucosal or gingival SCC in terms of overall survival (OS) and disease-free survival (DFS) rates. We also investigate whether the omission of adjuvant treatment represents an alternative option for the favorable group. We present the following article in accordance with the STROBE reporting checklist (available at https://dx.doi.org/10.21037/tro-21-1).

\section{Methods}

\section{Study design}

We conducted a retrospective study of 113 patients with primary buccal mucosal or gingival SCC who underwent surgical intervention with or without adjuvant treatment at Kaohsiung Medical University Chung-Ho Memorial Hospital from January 2010 to November 2018. The study was conducted in accordance with the Declaration of Helsinki (as revised in 2013). The study protocol was approved by the Institutional Review Board of Kaohsiung Medical University Chung-Ho Memorial Hospital (IRB number: KMUHIRB-E(I)-20200326) and individual consent for this retrospective analysis was waived. The standard surgical procedure was wide excision with unilateral or bilateral modified radical neck dissection. The study investigated two-dimensional radiation therapy, three-dimensional conformal radiation therapy, volumetric modulated arc therapy, and helical tomotherapy with doses ranged from 50 to 72 Gy. All patients received diagnoses of pT4aN0, stage IVA according to the seventh edition of the AJCC (4). Patients were excluded if they had received any neoadjuvant treatment, if their final pathologic histology was not SCC, if they had secondary oral cavity cancer, or if they had received prior treatment for head and neck cancer.

OS and DFS were analyzed as outcome variables. The definition of OS was the length of time from the date of diagnosis to death from any cause. The definition of DFS was the length of time from the last treatment date to any recurrent sign or symptom of cancer. Patients who were alive at the date of the last visit or observation were censored. Other variables included age, tumor size, pT4a type (bone or skin invasion), tumor cell differentiation, margin status [involved, close $(<5.0 \mathrm{~mm})$, or free $(\geq 5.0 \mathrm{~mm})$ ], presence of lymphovascular invasion (LVI), perineural invasion (PNI), and postoperative adjuvant treatment [radiotherapy (RT), chemotherapy, or combinedmodality therapy including chemoradiotherapy (CRT) or bioradiotherapy (Bio-RT)]. In addition, we defined the favorable group as patients without the involved margin, closed margin, LVI, PNI and poorly differentiated tumor cells. We also investigated these groups with and without adjuvant treatment in terms of DFS rate.

\section{Statistical analysis}

Kaplan-Meier survival curves were used to estimate OS and DFS rates by groups on the basis of baseline clinicopathological factors. Breslow tests (Generalized Wilcoxon) were applied for comparing survival curves between prognostic groups because these survival rates changed with time. We implemented multivariable analysis for prognostic factors for which $\mathrm{P}<0.1$, according to Breslow tests. A Cox proportional-hazards model was used to explore prognostic factors significantly associated with survival. Data were analyzed using SPSS Version 20.0 (IBM Statistics for Windows, Armonk, NY: IBM 


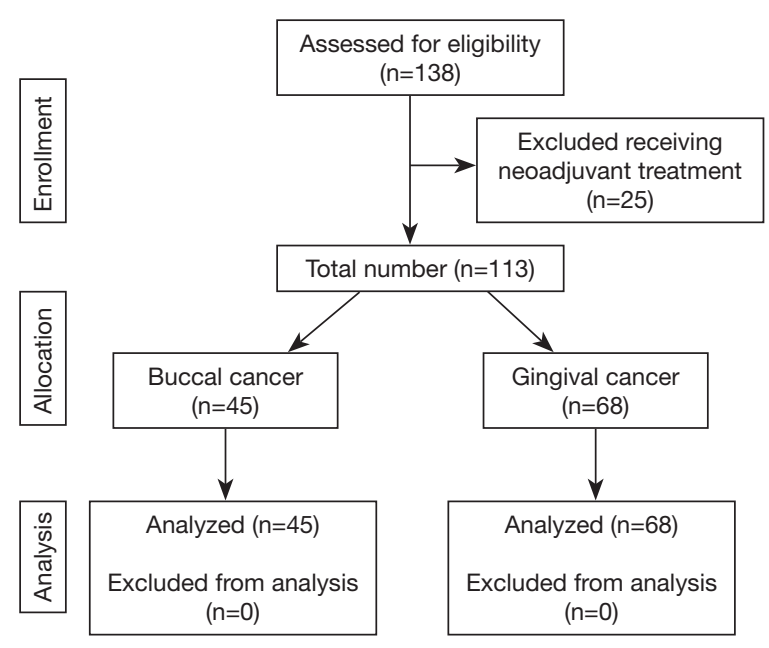

Figure 1 Flow of the patients through the study.

Corp.). A $P$-value of 0.05 was considered significant.

\section{Results}

In total, 113 patients were eligible for this study (Figure 1). The median follow-up duration was 38 months (range, 1-119 months). The mean age of patients was 55.67 years (range, 32-79 years), with a male-to-female ratio of 13.1:1. The demographic, clinical, and pathologic information of the study are summarized in Table 1. Forty-six patients received adjuvant combined therapy including CRT $(\mathrm{n}=45)$ and Bio-RT $(\mathrm{n}=1)$. Thirty-one patients received adjuvant RT. Thirty patients received no adjuvant treatment. The reason of no adjuvant therapy included multiple comorbidities, poor performance status, and personal considerations. The decision for adjuvant treatment was reached through multidisciplinary team discussion. RT was delivered at a conventional dose of 1.8-2 Gy/fraction for 5 days per week. The total dose ranged from 50 to $72 \mathrm{~Gy}$ and the RT technique included two-dimensional radiation therapy, three-dimensional conformal radiation therapy, volumetric modulated arc therapy, and helical tomotherapy. Triweekly or weekly cisplatin was used as a standard agent in the adjuvant CRT protocol. Cisplatin was administered on Day 1, 22, 43 in the triweekly group. The other regimen was to prescribe cisplatin weekly in order to reduce acute toxicity. A few patients $(\mathrm{n}=5)$ received oral uracil-tegafur or cetuximab (Erbitux) as alternative treatments. The reasons for cisplatin unsuitability were major cardiovascular diseases $(\mathrm{n}=1)$, poor kidney function $(\mathrm{n}=1)$ and personal refusal $(\mathrm{n}=3)$.

Median OS was 40 months (range, 1-123 months), and
Table 1 Patient characteristics ( $N=113)$

\begin{tabular}{|c|c|}
\hline Characteristics & Number (\%) \\
\hline \multicolumn{2}{|l|}{ Age (y) } \\
\hline Mean & 55.67 \\
\hline Median & 55.00 \\
\hline \multicolumn{2}{|l|}{ Gender } \\
\hline Male & 105 (92.92) \\
\hline Female & $8(7.08)$ \\
\hline \multicolumn{2}{|l|}{ Laterality } \\
\hline Right & $66(58.41)$ \\
\hline Left & 47 (41.59) \\
\hline \multicolumn{2}{|l|}{ Primary site } \\
\hline Buccal & 45 (39.82) \\
\hline Lower gingiva & $59(52.21)$ \\
\hline Upper gingiva & $9(7.96)$ \\
\hline \multicolumn{2}{|l|}{ T4a type } \\
\hline Bone invasion & 81 (71.68) \\
\hline Skin invasion & $23(20.35)$ \\
\hline Both & $9(7.96)$ \\
\hline \multicolumn{2}{|l|}{ Differentiation } \\
\hline Well & $77(68.14)$ \\
\hline Moderate & $33(29.20)$ \\
\hline Poor & $3(2.65)$ \\
\hline \multicolumn{2}{|c|}{ Lymphovascular invasion } \\
\hline Negative & 105 (92.92) \\
\hline Positive & $8(7.08)$ \\
\hline \multicolumn{2}{|l|}{ Perineural invasion } \\
\hline Negative & $95(84.07)$ \\
\hline Positive & 18 (15.93) \\
\hline \multicolumn{2}{|l|}{ Margin } \\
\hline Involved & $20(17.70)$ \\
\hline Close & $42(37.17)$ \\
\hline Free & $51(45.13)$ \\
\hline \multicolumn{2}{|l|}{ Adjuvant treatment } \\
\hline None & $30(26.55)$ \\
\hline Radiotherapy & $31(27.43)$ \\
\hline Chemotherapy & $6(5.31)$ \\
\hline Combined therapy & $46(40.71)$ \\
\hline
\end{tabular}




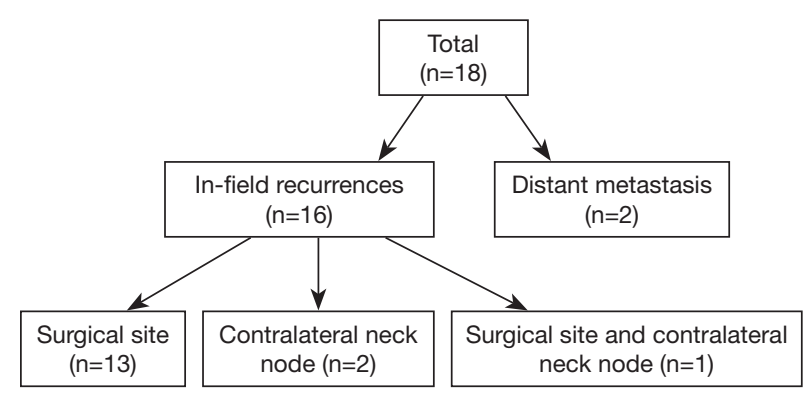

Figure 2 Failure patterns of the study patients $(n=18)$.

the median DFS was 38 months (range, 1-123 months). In the entire study, 5-year OS and DFS rates were $73.7 \%$ and $83.6 \%$, respectively, and 18 recurrences $(15.9 \%)$ and 27 deaths (23.9\%) occurred. Of all recurrences (Figure 2), 13 patients had primary surgical site recurrence $(11.5 \%)$, 2 patients had contralateral neck node recurrence (1.8\%), and 1 patient had both primary site and contralateral node recurrence $(0.9 \%)$. In addition, 2 patients had distant metastasis (1.8\%); 1 had lung metastasis; and the other had multiple lung, bone, and adrenal metastases. On univariate analysis, LVI, PNI, and margin status exerted significant effects on the DFS rate, and tumor cell differentiation exerted significant effects on the OS rate (Table 2). According to multivariate Cox regression analysis, the margin status was a significant factor affecting the DFS rate [odds ratio $(\mathrm{OR})=5.036,95 \%$ CI: 1.243-20.397, $\mathrm{P}=0.038$, Table 2].

As per the aforementioned result, we defined patients without the involved margin, close margin, PNI, LVI, and poorly differentiated tumor cells as the favorable group $(n=38)$. The unfavorable group $(n=75)$ included patients with the involved margin, close margin, PNI, LVI, or poorly differentiated tumor cells. We further analyzed DFS and OS rates of the two subgroups. The 5-year OS rates of the favorable and unfavorable groups were $75.3 \%$ and $72.7 \%$, respectively. The 5 -year DFS rates of the favorable and unfavorable groups were $94.7 \%$ and $77.8 \%$, respectively. The favorable group had a better DFS (OR: $4.881,95 \%$ CI: 1.060-22.482, $\mathrm{P}=0.023)$, but did not have a significant effect on OS (OR: 1.272, 95\% CI: 0.498-3.249, P=0.544) (Figure 3).

In addition, we explored the impact of adjuvant therapy in these two groups. We divided the favorable patients into two groups: with (Group B, n=24) or without (Group A, n=14) adjuvant therapy; the unfavorable patients were also divided into two groups: one group with (Group C, $\mathrm{n}=59$ ) adjuvant therapy and the other group without (Group D, n=16) adjuvant therapy. The 5 -year OS rates of groups A, B, C, and $\mathrm{D}$ were $78.6 \%, 72.4 \%, 71.8 \%$, and $79.4 \%$, respectively. The 5 -year DFS rates of groups A, B, C, and D were $100 \%, 91.5 \%, 79.7 \%$, and $69.6 \%$, respectively (Figure 4). No significant difference in the OS rate (OR: 1.036, 95\% CI: $0.207-5.198, \mathrm{P}=0.643$ ) was observed between groups A and $\mathrm{B}$. Because no recurrent cases occurred within group A, statistical methods could not be used to analyze DFS rate between group A and B. Within the favorable group of patients without any adjuvant treatment (Group A, $n=14$ ), three deaths and no recurrent cases occurred during followup (median 53 months, range 15-111 months). Within the favorable group of patients with adjuvant treatment (Group B, n=24), five deaths included three deaths from medical infections and two deaths from liver cancer. Two recurrent cases occurred during follow-up (median 33 months, range 8-119 months). The two relapse patterns were local recurrence involving masticator and pterygoid spaces and lung metastasis, respectively. Within the patients without adjuvant treatment (Group C and Group D), two recurrent cases underwent salvage operation and two recurrent cases received salvage CRT.

\section{Discussion}

This is the first study in which data exclusively from groups with buccal or gingival SCC were collected for detailed survival analysis. In our clinical observations, buccal mucosal or gingival cancer often presented locally and in the advanced stage. The tumor invades the surrounding tissue early in the development of the disease, such as cortical bone. Consequently, these groups may be upstaged to pT4a. According to AJCC (4), the same prognostic stage is used regardless of the primary oral tumor location. Whether the tumor subsite of OCSCC is a prognostic factor is controversial, although buccal and gingival SCC exhibited no significant difference in survival results in our study. Multiple studies have published data suggesting that the gingival subsite is a favorable prognostic feature in OCSCC (8-10). However, some studies conducted in Western countries have shown that the subsite for buccal SCC is associated with more aggressive OCSCC. Even if early-stage buccal SCC was treated post-operatively, a higher recurrent rate was still observed (11-14). Camilon et al. showed that, with unmatched data, buccal cancer had significantly poorer OS and disease-specific survival (DSS) than did cancers elsewhere in the oral cavity $(\mathrm{P}<0.001)$. After case matching, the differences between OS and DSS 
Table 2 Factors affecting DFS and OS (N=113)

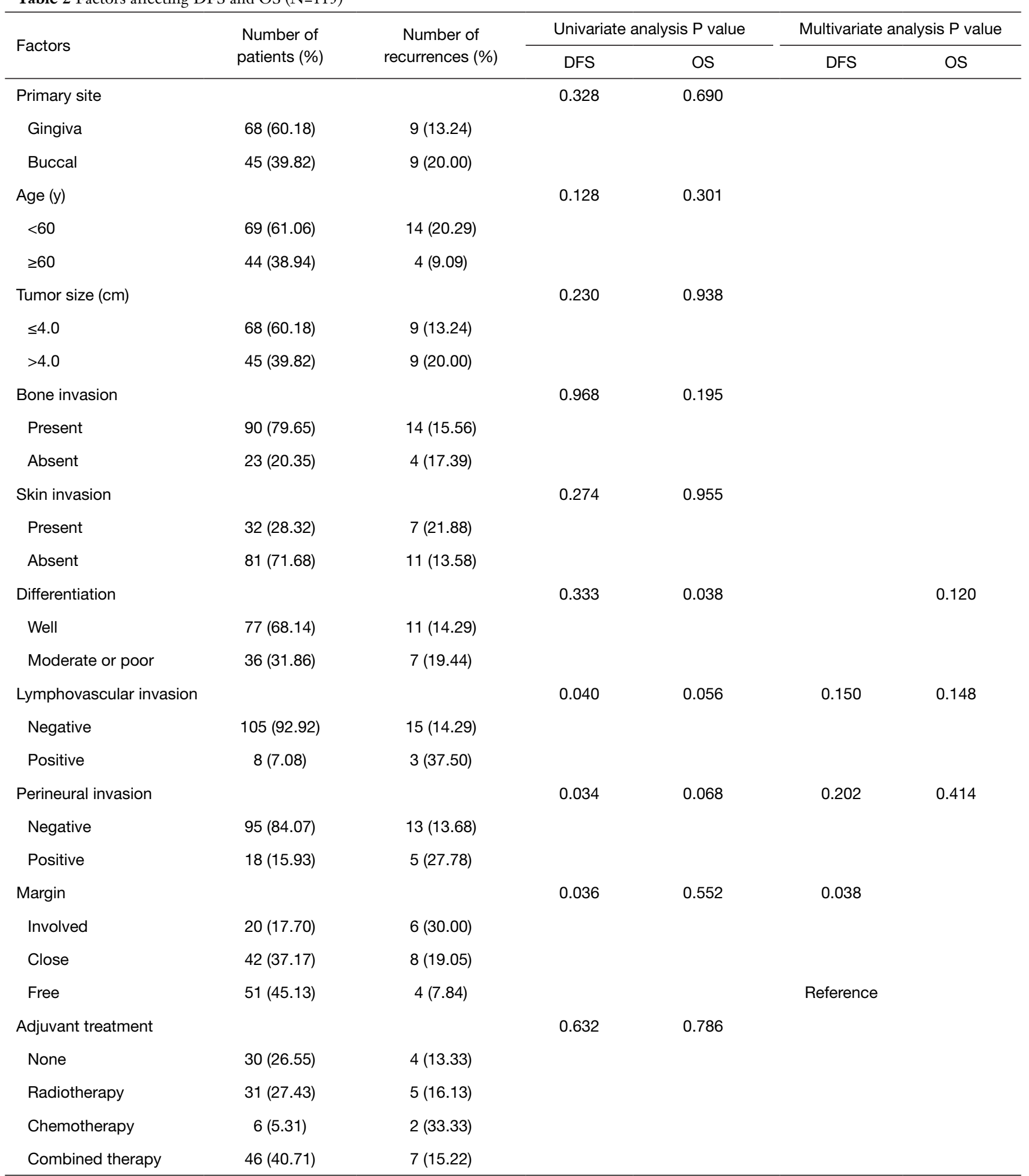



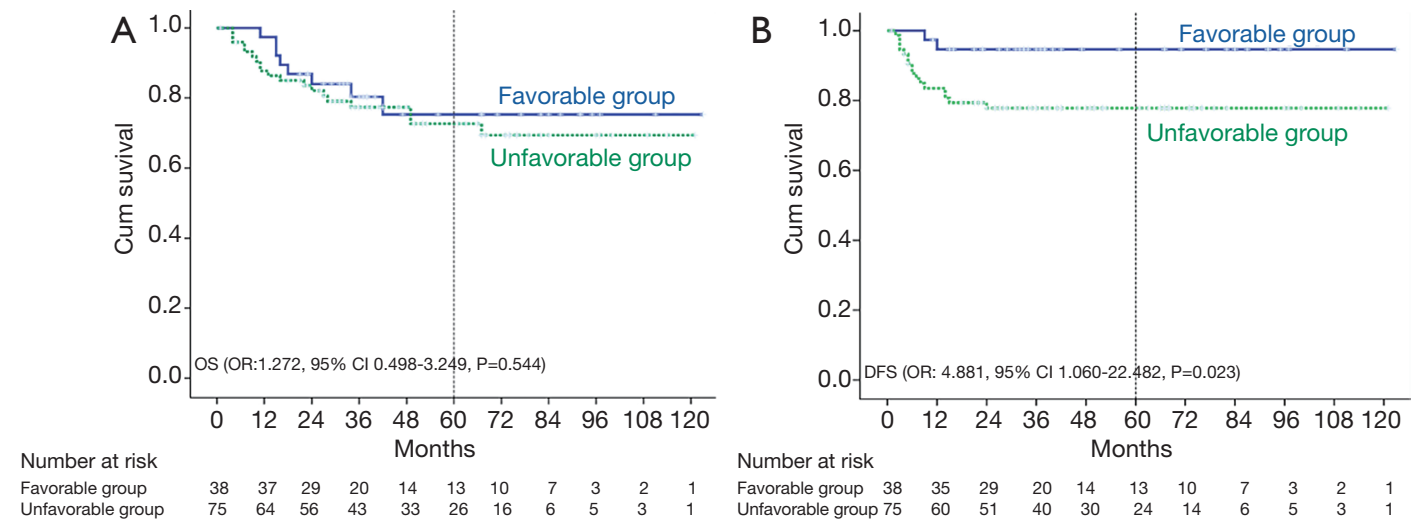

Figure 3 Kaplan-Meier survival analysis for patients in the favorable and unfavorable groups: (A) overall survival and (B) disease-free survival.

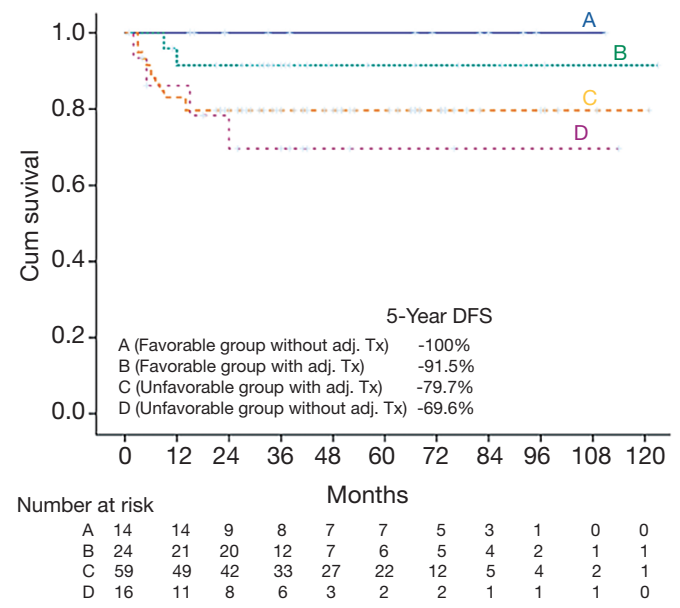

Figure 4 Kaplan-Meier curve showing disease-free survival in subgroups. Adj. Tx, adjuvant treatment.

for buccal cancer versus for nonbuccal oral cancer were no longer significant (44\% vs. 48\%, $\mathrm{P}=0.113$ ) (15). However, a study conducted using the data from the Taiwanese Cancer Registry Database showed a statistically significantly higher 5-year DSS and OS for buccal SCC than for oral tongue SCC (HR: 1.08; 95\% CI: 1.01-1.15, $\mathrm{P}=0.0297$ and HR: 1.07 ; 95\% CI: $1.01-1.13, \mathrm{P}=0.0231$, respectively). Despite the higher prevalence of $\mathrm{pT} 4$ and stage IV, a lower prevalence of $\mathrm{pN} 2$ diseases was found for buccal SCC $(15.2 \%$ vs. $18.5 \%, \mathrm{P}<0.0001)(2)$.

Among patients with pT4aN0, our result is not inferior to those from other studies. In our entire study, 5-year OS and DFS rates were $73.7 \%$ and $83.6 \%$, respectively. Kirke et al. demonstrated that 3-year OS rate was $67.5 \%$ for adjuvant RT and $70.5 \%$ for adjuvant CRT, respectively, but the primary tumor subsites of patients with pT4aN0 vary and include the oral cavity, oropharynx, larynx and hypopharynx (16). Namin et al. showed that the 5-year OS rate was $57 \%$ in those with adjuvant RT and $44 \%$ in those without adjuvant RT. The primary subsite of pT4aN0 included the oral cavity such as the tongue, floor of mouth, gingival, buccal, and retromolar trigones. Subgroup analysis demonstrated that the tongue and floor-of-mouth subsites are significantly associated with worse OS in pT4aN0 OCSCC regardless of univariate and multivariable analysis results (17). The reason for this difference in survival rates among patients with $\mathrm{pT} 4 \mathrm{aN} 0$ is the heterogeneous nature of the cancer and the large range of outcomes. The specific tumor location in the oral cavity might be more accurately analyzed with the same tumor and nodal classification.

There was a similar study exploring the necessity of adjuvant treatment for only the pT4a independent factor. Nassiri et al. concluded that, among 40 patients with pT4N0 mandibular gingival SCC, excellent local control and survival rates resulted from with surgery alone. The result of 5 -year OS and DFS was $80.6 \%$ and $84.5 \%$, respectively (8). However, the limitations of this study were small sample size and a lack of detailed histologic analysis. Because of the excellent results from similar study and our subgroup analysis, data suggest that the omission of adjuvant treatment for favorable patients may be considered as an alternative option. Because Patel et al. demonstrated that factors predicting survival were diverse and complex, clinicians should consider age and comorbidity among patients with OCSCC (18). Adjuvant therapy may have resulted in more treatment-induced severe toxicities particularly in older patients with poor performance status 
or more comorbidity. This represents a dilemma in clinical decision making. Consequently, the omission of adjuvant therapy may be considered for favorable patients potentially unable to tolerate it.

In our study, LVI, PNI, and margin status had significant effects on DFS, and tumor cell differentiation had a significant effect on OS according to univariate analysis. We used these significant pathologic factors to distinguish favorable and unfavorable groups. Prateek at el. showed that the clinicopathologic determinants of outcome in pT4a gingivobuccal SCC were LVI, PNI, cervical nodal metastasis, and extracapsular nodal extension (5). Because regional nodal metastasis was excluded in our research, the predictors and prognostic influence could be more accurately ascertained from patients with pT4aN0. In addition, margin status was the only significant factor affecting DFS according to multivariate Cox regression analysis. Adequate pathological margins played a crucial role in achieving satisfactory local control. The definitions of adequate margins generally vary depending on several factors such as the primary tumor location of head and neck cancers. According to the systematic review, the definition of a close margin was considered to range from 2 to $7 \mathrm{~mm}$ in oral cavity cancer (19). In addition, Singh et al. claimed that the mean and median spread of tumors were 8.6 and $7 \mathrm{~mm}$, respectively, in patients with pT4 OCSCC. They recommended an adequate margin of $15 \mathrm{~mm}$ from the visualized disease in the bone or mucosa (20). In our study, a clear margin is defined as $5 \mathrm{~mm}$ or more from the resected margin per National Comprehensive Cancer Network guidelines (21).

The most lymph nodes metastasis for buccal or gingival SCC are at ipsilateral level I or II (22). Lymph node metastasis occurred in $3(2.7 \%)$ of all patients in our study. Two cases had one positive node at the contralateral level IIA, and one case had positive nodes at the contralateral levels II, III, and IV. Because all three patients underwent elective ipsilateral nodal radiotherapy, no pattern of ipsilateral nodal recurrence was observed. Moreover, a review of the medical reports of two metastatic patients indicated that the primary tumor sizes were bulky 91 and $75 \mathrm{~mm}$, respectively. One of the depth of invasion (DOI) measurements was $54 \mathrm{~mm}$, and the other was not described in the medical record. Due to low probability of distant metastasis, studies in head and neck patients are generally limited by small sample sizes, particularly factors impacting on distant metastasis (23). In theory, larger tumors or deeper invasions are more likely to have micrometastases.
More research is required on these topics.

Limitations of this study include its retrospective design with small sample-size within subgroup analysis. Second, a few patients were lost to follow-up prior to the completion of the cancer surveillance period. Finally, some clinicopathologic disease characteristics were absent for analysis, such as DOI.

\section{Conclusions}

Although current guidelines recommend that $\mathrm{pT} 4 \mathrm{a}$ is an independent factor for adjuvant therapy in patients with OCSCC, this study demonstrates that a subset (favorable group) of pT4aN0 buccal mucosal or gingival SCC had fair DFS rate without adjuvant therapy. Therefore, the omission of adjuvant therapy may be considered for this subgroup.

\section{Acknowledgments}

Funding: None.

\section{Footnote}

Reporting Checklist: The authors have completed the STROBE reporting checklist. Available at https://dx.doi. org/10.21037/tro-21-1

Conflicts of Interest: All authors have completed the ICMJE uniform disclosure form (available at https://dx.doi. org/10.21037/tro-21-1). CJH serves as an unpaid editorial board member of Therapeutic Radiology and Oncology from May 2020 to Apr 2022. The other authors have no conflicts of interest to declare.

Ethical Statement: The authors are accountable for all aspects of the work in ensuring that questions related to the accuracy or integrity of any part of the work are appropriately investigated and resolved. The study was conducted in accordance with the Declaration of Helsinki (as revised in 2013). The study protocol was approved by the Institutional Review Board of Kaohsiung Medical University Chung-Ho Memorial Hospital (IRB number: KMUHIRB-E(I)-20200326) and individual consent for this retrospective analysis was waived.

Open Access Statement: This is an Open Access article distributed in accordance with the Creative Commons Attribution-NonCommercial-NoDerivs 4.0 International 
License (CC BY-NC-ND 4.0), which permits the noncommercial replication and distribution of the article with the strict proviso that no changes or edits are made and the original work is properly cited (including links to both the formal publication through the relevant DOI and the license). See: https://creativecommons.org/licenses/by-nc-nd/4.0/.

\section{References}

1. Taiwan Cancer Registry Annual Report. 2017. Available online: https://www.hpa.gov.tw/EngPages/Index.aspx

2. Liao CT, Wen YW, Yang LY, et al. Comparative clinical outcomes of Taiwanese patients with resected buccal and tongue squamous cell carcinomas. Oral Oncol 2017;67:95-102.

3. Liao CT, Huang SF, Chen IH, et al. Tongue and buccal mucosa carcinoma: is there a difference in outcome? Ann Surg Oncol 2010;17:2984-91.

4. Edge SB, Byrd DR, Compton CC, et al. AJCC Cancer staging manual. 7th ed. New York: Springer Science + Business Media, 2010:29-40.

5. Jain PV, Sharan R, Manikantan K, et al. Clinicopathologic Determinants of Outcome in Pathologic T4a (pT4a) Squamous Cell Carcinoma of the Gingivobuccal Subsite of the Oral Cavity. Indian J Surg Oncol 2019;10:594-9.

6. Cooper JS, Pajak TF, Forastiere AA, et al. Postoperative concurrent radiotherapy and chemotherapy for high-risk squamous-cell carcinoma of the head and neck. N Engl J Med 2004;350:1937-44.

7. Bernier J, Domenge C, Ozsahin M, et al. Postoperative irradiation with or without concomitant chemotherapy for locally advanced head and neck cancer. N Engl J Med 2004;350:1945-52.

8. Nassiri AM, Campbell BR, Mannion K, et al. Survival Outcomes in T4aNOM0 Mandibular Gingival Squamous Cell Carcinoma Treated with Surgery Alone. Otolaryngol Head Neck Surg 2019;160:870-5.

9. Niu LX, Feng ZE, Wang DC, et al. Prognostic factors in mandibular gingival squamous cell carcinoma: A 10year retrospective study. Int J Oral Maxillofac Surg 2017;46:137-43.

10. Okura M, Yanamoto S, Umeda M, et al. Prognostic and staging implications of mandibular canal invasion in lower gingival squamous cell carcinoma. Cancer Med 2016;5:3378-85.

11. Lubek JE, Dyalram D, Perera EH, et al. A retrospective analysis of squamous carcinoma of the buccal mucosa: an aggressive subsite within the oral cavity. J Oral Maxillofac Surg 2013;71:1126-31.
12. Diaz EM Jr, Holsinger FC, Zuniga ER, et al. Squamous cell carcinoma of the buccal mucosa: one institution's experience with 119 previously untreated patients. Head Neck 2003;25:267-73.

13. Sieczka E, Datta R, Singh A, et al. Cancer of the buccal mucosa: are margins and T-stage accurate predictors of local control? Am J Otolaryngol 2001;22:395-9.

14. Mishra RC, Singh DN, Mishra TK. Post-operative radiotherapy in carcinoma of buccal mucosa, a prospective randomized trial. Eur J Surg Oncol 1996;22:502-4.

15. Camilon PR, Stokes WA, Fuller CW, et al. Does buccal cancer have worse prognosis than other oral cavity cancers? Laryngoscope 2014;124:1386-91.

16. Kirke DN, Qureshi MM, Kamran SC, et al. Role of adjuvant chemoradiotherapy in T4N0 stage IV head and neck cancer: A National Cancer Database analysis. Head Neck 2018;40:1174-84.

17. Namin AW, Bollig CA, Harding BC, et al. Implications of Tumor Size, Subsite, and Adjuvant Therapy on Outcomes in pT4aN0 Oral Cavity Carcinoma. Otolaryngol Head Neck Surg 2020;162:683-92.

18. Patel KB, Martin D, Zhao S, et al. Impact of age and comorbidity on survival among patients with oral cavity squamous cell carcinoma. Head Neck 2021;43:268-77.

19. Alicandri-Ciufelli M, Bonali M, Piccinini A, et al. Surgical margins in head and neck squamous cell carcinoma: what is 'close'? Eur Arch Otorhinolaryngol 2013;270:2603-9.

20. Singh A, Mair M, Singhvi H, et al. Incidence, predictors and impact of positive bony margins in surgically treated $\mathrm{T} 4$ stage cancers of the oral cavity. Oral Oncol 2019;90:8-12.

21. National Comprehensive Cancer Network. Oral cavity cancer (Version 2.2020). Available online: https://www.nccn. org/professionals/physician_gls/pdf/head-and-neck.pdf

22. Woolgar JA. Histological distribution of cervical lymph node metastases from intraoral/oropharyngeal squamous cell carcinomas. Br J Oral Maxillofac Surg 1999;37:175-80.

23. Aires FT, Lin CS, Matos LL, et al. Risk Factors for Distant Metastasis in Patients with Oral Cavity Squamous Cell Carcinoma Undergoing Surgical Treatment. ORL J Otorhinolaryngol Relat Spec 2017;79:347-55.

doi: 10.21037/tro-21-1

Cite this article as: Chao TY, Chan LP, Lee HH, Kao YH, Jhong YP, Chiang CH, Chuang ST, Huang CJ. Is pathological T4a an independent indication of adjuvant therapy in buccal mucosal or gingival squamous cell carcinoma? Ther Radiol Oncol 2021;5:12. 